\title{
The Role of Autophagy in Hepatitis C Virus Infection
}

\author{
Jun Cheng, Min Quan, Liang Wu and Qi Wang
}

\begin{abstract}
Hepatitis $\mathrm{C}$ virus (HCV) infects $\approx 2 \%$ of the world's population. HCV infection not only causes acute and chronic hepatitis, but also leads to liver cirrhosis and hepatocellular carcinoma (HCC). The molecular pathogenesis of HCV infection has been explored and many evidence indicated that autophagy is an important process for its life cycle, although autophagy was thought as a mechanism to eliminate invaded HCV from hepatocyte. Structural and non-structural proteins of HCV are important regulators of autophagy, and HCV uses autophagy as a necessary step in its replication. Down-regulation of innate immune response by HCV through unfolded protein response (UPR) and autophagy induction was used as a pathway to establish chronic HCV infection in the liver. Meanwhile, the infected hepatocyte is also using autophagy mechanism to eradicate HCV virus from liver. The study on relationship between HCV and autophagy will pave the new way to understand HCV life cycle and to find new strategy for prevention and treatment of liver diseases caused by HCV infection.
\end{abstract}

Key words: Autophagy; Hepatitis C virus; Liver; Viral replication; Innate immunity

A utophagy is a process which helps to maintain cellular energy homeostasis and remove damaged organelles. ${ }^{1-3}$ Autophagy research has been recently gained momentum as it is increasingly being recognized to play fundamental roles in diverse aspects of human pathophysiology including virus infection and its subsequent complications. ${ }^{4-10}$ In the liver, autophagy is an adaptive process that is activated in response to stress including acute and chronic inflammation, including viral infection. ${ }^{11-19}$ Autophagy is also a conserved degradative pathway used as a host defense mechanism against intracellular pathogens. Hepatitis C virus (HCV) can evade or subvert autophagy by escaping the innate immune response to insure their replication. ${ }^{20-23} \mathrm{HCV}$ infection is a major cause of chronic liver disease and liver cancer in the world. ${ }^{24}$ Infection with the HCV genotype 1 in particular is considered a clinical risk factor for the development of hepatocellular carcinoma, although the molecular mechanisms of the pathogenesis are largely unknown. ${ }^{25}$ Recently there were several reviews on the autophagy in the liver infected with $\mathrm{HCV}^{26-30}$ Nevertheless, the molecular details of viral interaction with autophagy remain largely unknown.

\section{EVIDENCE THAT HEPATITIS C VIRUS REPLICATION IS RELATED TO AUTOPHAGY}

Viral infection induces autophagy, which can promote cell survival by removing the stress inducer or by attenuating

\footnotetext{
Correspondence: Jun Cheng, No. 8 Jingshun Street East, Chaoyang District, Beijing 100015, China;

E-mail: jun.cheng.ditan@gmail.com
}

its dangerous effects. High levels of autophagy occur during infection of cells with HCV. Vescovo et al. analyze the level of autophagy in liver biopsy samples from patients with $\mathrm{HCV}$ infection. ${ }^{31}$ They found that there was an inverse correlation between microvesicular steatosis and level of autophagy. HCV selectively induced autophagy of lipids in virus-infected and replicon cells. In each system, autophagosomes frequently colocalized with lipid deposits, mainly formed by unesterified cholesterol. Inhibition of the autophagic process in these cells significantly increased the induction of cholesterol accumulation by HCV. This study confirmed the counteractions between autophagy and alterations in lipid metabolism induced by HCV.

The relations between autophagy and HCV infection were also indicated in the study of the liver steatosis from transgenic mice expressing the $\mathrm{HCV}$ polyprotein. Nishina et al. found fat accumulation in the liver of mice expressing the HCV polyprotein. ${ }^{32}$ Hepatic iron levels were positively correlated with triglyceride concentrations in the liver. The livers of transgenic mice showed a lower expression of carnitine palmitoyl transferase I, a higher expression of sterol-regulatory element-binding protein (SREBP) 1 and fatty acid synthetase (FASN) and an activated unfolded protein response (UPR) indicated by a higher expression of unspliced and spliced X-box DNA-binding protein 1 (XBP-1), phosphorylated eukaryotic initiation factor-2 $\alpha$ (p-eIF2 $\alpha$ ), CCAAT/enhancer-binding protein homology protein (CHOP) and abundant autophagosomes. Treatment with the anti-oxidant $\mathrm{N}$-acetyl cysteine (NAC) dramatically reduced hepatic steatosis in transgenic mice through decreased expression of unspliced and spliced XBP-1, p-eIF2 $\alpha$, and CHOP. So in this study, the iron-induced UPR appears to be one 
of the mechanisms responsible for fat accumulation in the liver in transgenic mice expressing the HCV polyprotein.

Ultrastructural and biochemical results demonstrated that non-structural proteins of HCV co-localized with key proteins involved in autophagy regulations. HCV induces changes in the host cell's membranes, resulting in a membranous web. The non-structural proteins of the viral replication complex are thought to be associated with these newly synthesized membranes. Ferraris et al studied this phenomenon, using a Huh7.5 cell clone displaying high levels of replication of a subgenomic replicon of the JFH-1 strain. ${ }^{33}$ Electron microscopy of ultrathin sections of these cells revealed the presence of numerous double membrane vesicles (DMVs). These structures were highly specific to HCV. DMVs were found to contain the NS3 and NS5A proteins, HCV RNA and LC3-II proteins. HCV may induce DMVs by activating the autophagy pathway. This could represent a strategy to conceal the viral RNA and help the virus to evade double-stranded RNA-triggered host antiviral responses.

$\mathrm{HCV}$ proteins are playing very important roles in autophagy regulation. Chu et al. demonstrated that persistent expression of HCV non-structural proteins leads to increased autophagy and mitochondrial injury in human hepatoma cells. Increased reactive oxygen species (ROS) generation in the mitochondria, mitochondrial injury and degeneration, and increased lipid accumulation were common among all HCV protein-expressing cells regardless of whether they expressed the structural or non-structural proteins. Expression of the non-structural proteins also led to increased oxidative stress in the cytosol, membrane blebbing in the endoplasmic reticulum, and accumulation of autophagocytic vacuoles. Alterations of cellular redox state, on the other hand, significantly changed the level of autophagy, suggesting a direct link between oxidative stress and HCV-mediated activation of autophagy.

Ait-Goughoulte et al. found that HCV infection induces autophagic vacuole formation in $\mathrm{IHH}$ using electron microscopy. Fluorescence microscopy displayed localization of autophagic markers, microtubule-associated protein-1 light chain-3 (LC-3) and Apg5, on the vacuoles of HCV-infected hepatocytes. The results suggested that HCV genotype 1a (clone H77) can be serially passaged in $\mathrm{IHH}$ and that $\mathrm{HCV}$ infection induces an autophagic response in hepatocytes.

\section{REGULATION OF AUTOPHAGY BY HEPATITIS C VIRUS INFECTION}

Structural and non-structural proteins of HCV have been demonstrated for their effects on autophagy in hepatocyte. ${ }^{34}$ Among them, NS4B is an important viral protein for regulation of hepatocyte autophagy. Su et al. found NS4B protein is playing a role in autophagy regulation. Rab5 and class III phosphoinositide 3-kinase (PI3K) Vps34 are involved in NS4B-induced autophagy. HCV NS4B alone can induce autophagy signaling; amino acid residues 1 to 190 of NS4B are sufficient for this induction. Inhibition of Rab5 function resulted in significant reduction of NS4B- or $\mathrm{HCV}$-induced autophagic vesicle formation. Moreover, the autophagy induction was impaired by inhibition of class III phosphoinositide 3-kinase (PI 3-kinase) Vps34 function. Coimmunoprecipitation assay indicated that NS4B formed a complex with Rab5 and Vps34, supporting the notion that Rab5 and Vps34 are involved in NS4B-induced autophagy.

Autophagy protein ATG5 interacts transiently with the HCV NS5B protein early during infection and induces autophagy of infected hepatocytes. ATG5 initiates the formation of DMVs. Upon infection, DMVs have been shown to harbor the replicase complex of $\mathrm{HCV}$. Guévin et al identified ATG5 as an interacting protein for the HCV NS5B. ${ }^{35}$ ATG5 protein colocalizes with NS4B, immunofluorescence staining demonstrated a strong colocalization of ATG5 and NS5B. However, colocalization was completely lacking at 5DPI, suggesting that HCV utilizes ATG5 as a proviral factor during the onset of viral infection.

The non-structural protein NS3/4A of $\mathrm{HCV}$ is a serine protease which can enzymatically digest two host cellular proteins, mitochondria-associated antiviral signaling protein (MAVS) and Toll/interleukin-1 receptor domain containing adaptor inducing IFN- $\beta$ (TRIF) to block retinoic acid-inducible gene (RIG) I-mediated interferon (IFN) responses. Since these two proteins are the adaptor molecules in the RIG-I and TLR3 pathways, respectively, their cleavage has been suggested as a pivotal mechanism by which HCV blunts the IFN- $\alpha / \beta$ signaling and antiviral responses. Desai et al generated transgenic mice that express the HCV NS3/4A proteins. ${ }^{36}$ They evaluated the effects of $\mathrm{HCV}$ serine protease on the innate immune responses and their interactions. Expression of $\mathrm{HCV}$ NS3/4A resulted in cleavage of intrahepatic MAVS. Induced production of IFN $\alpha$ and $\beta$ resulted in different autophagic responses.

Immunity-associated GTPase family M (IRGM) is a target of HCV that subvert the autophagy network. Grégoire et al determined the ability of 83 proteins of several families of RNA viruses, to interact with 44 human autophagy-associated proteins using yeast two-hybrid and bioinformatic analysis. ${ }^{37}$ IRGM was found to interact with ATG5, ATG10, MAP1CL3C and SH3GLB1. Reduction of IRGM expression using small interfering RNA impairs HCV-induced autophagy and viral particle production. Moreover, IRGM and HCV NS3 proteins interaction per se is sufficient to induce autophagy, through an IRGM dependent pathway. 
These result reveals an unexpected role of IRGM in HCV-induced autophagy and suggests that HCV may use this strategy to manipulate autophagy to improve $\mathrm{HCV}$ infectivity.

In the neuropathogenesis of patients with HIV/ $\mathrm{HCV}$ co-infection, recent results indicated that autophagy induced by HCV core protein is a key step to potentiate of human immunodeficiency virus-1 (HIV-1) neurotoxicity. Vivithanaporn et al investigate $\mathrm{HCV}$ infectivity and effects on neuronal survival and neuroinflammation in conjunction with HIV infection. ${ }^{10}$ Human microglia, astrocyte and neuron cultures were infected with cell culture-derived $\mathrm{HCV}$ or exposed to HCV core protein with or without HIV-1 infection or HIV-1 viral protein R (Vpr) exposure. Host immune gene expression and cell viability were measured. Patch-clamp studies of human neurons were performed in the presence or absence of $\mathrm{HCV}$ core protein. Neurobehavioral performance and neuropathology were examined in HIV-1 Vpr-transgenic mice in which stereotaxic intrastriatal implants of $\mathrm{HCV}$ core protein were performed. HCV-encoded RNA as well as HCV core and NS3 proteins were detectable in human microglia and astrocytes infected with $\mathrm{HCV}$. $\mathrm{HCV}$ core protein exposure induced expression of pro-inflammatory cytokines including interleukin-1 $\beta$, interleukin- 6 and tumor necrosis factor- $\alpha$ in microglia, but not in astrocytes while increased chemokine (e.g. CXCL10 and interleukin-8) expression was observed in both microglia and astrocytes. HCV core protein modulated neuronal membrane currents and reduced both $\beta$-III-tubulin and lipidated LC3-II expression. Neurons exposed to supernatants from HCV coreactivated microglia exhibited reduced $\beta$-III-tubulin expression. HCV core protein neurotoxicity and interleukin-6 induction were potentiated by HIV-1 Vpr protein. HIV-1 Vpr transgenic mice implanted with HCV core protein showed gliosis, reduced neuronal counts together with diminished LC3 immunoreactivity. HCV core-implanted animals displayed neurobehavioral deficits at days 7 and 14 post-implantation. HCV core protein exposure caused neuronal injury through suppression of neuronal autophagy in addition to neuroimmune activation. The additive neurotoxic effects of HCV- and HIV-encoded proteins highlight extrahepatic mechanisms by which $\mathrm{HCV}$ infection worsens the disease course of HIV infection.

\section{UNFOLDED PROTEIN RESPONSE IS INVOLVED IN HCV-INDUCED AUTOPHAGY}

Different physiological and pathological situations that produce alterations in the endoplasmic reticulum, lead to a condition known as ER stress. UPR is tailored essentially to reestablish ER homeostasis. However, when persistent, ER stress can switch the cytoprotective functions of UPR into cell death promoting mechanisms. One of the cellular mechanisms that are regulated by ER stress is autophagy. The UPR is an ensemble of signal transduction pathways that respond to perturbations in the oxidative, pro-folding environment of the endoplasmic reticulum.

Sir et al. demonstrated that HCV can suppress autophagic protein degradation by suppressing the fusion between autophagosomes and lysosomes. ${ }^{38}$ This suppression causes the accumulation of autophagosomes and enhances HCV RNA replication. Further analysis indicated that the induction of autophagosomes by HCV is dependent on the UPR. The studies thus delineate a molecular pathway by which HCV induces autophagosomes. The prolonged perturbation of the autophagic pathway by HCV likely plays an important role in $\mathrm{HCV}$ pathogenesis.

Sir et al. demonstrates by a battery of morphological and biochemical assays that $\mathrm{HCV}$ induces the accumulation of autophagosomes in cells without enhancing autophagic protein degradation. ${ }^{38}$ This induction of autophagosomes depended on the UPR, as the suppression of UPR signaling pathways suppressed HCV-induced lipidation of LC3, a necessary step for the formation of autophagosomes. The suppression of UPR or the suppression of expression of LC3 or Atg7, a protein that mediates LC3 lipidation, suppressed HCV replication, indicating a positive role of UPR and the incomplete autophagic response in HCV replication. The studies delineate the molecular pathway by which HCV induces autophagic vacuoles and also demonstrate the perturbation of the autophagic response by $\mathrm{HCV}$. These unexpected effects of $\mathrm{HCV}$ on the host cell likely play an important role in $\mathrm{HCV}$ pathogenesis.

\section{M PACT O F THE A U T OPHA GY MACHINERY ON HEPATITIS C VIRUS INFECTION}

Autophagy is involved in the degradation of cellular organelles and the elimination of invasive microorganisms. On the other hand, HCV exploits the autophagy pathway for viral propagation. In cell culture, autophagy proteins are proviral factors that favor initiation of $\mathrm{HCV}$ infection. Autophagy proteins are required for translation of incoming viral RNA. Dreux et al propose that autophagy factors might support the delivery of incoming RNA to the translation apparatus and/or the recruitment of cellular factors required to initiate $\mathrm{HCV}$ translation. ${ }^{39}$

Taguwa et al. show that replication of HCV RNA induces autophagy to inhibit cell death. ${ }^{40}$ Cells harboring an HCV replicon RNA of genotype $1 b$ strain Con 1 but not of genotype 2a strain JFH1 exhibited an incomplete acidification of the autolysosome due to a 
lysosomal defect, leading to the enhanced secretion of immature cathepsin B. The suppression of autophagy in the Con $1 \mathrm{HCV}$ replicon cells induced severe cytoplasmic vacuolation and cell death. These results suggest that HCV harnesses autophagy to circumvent the harmful vacuole formation and to maintain a persistent infection.

Autophagic autolysosome maturation was required to promote HCV RNA replication and that it did so by suppressing innate antiviral immunity. The molecular mechanisms of assembly and budding of HCV is closely related to autophagy. The budding of several enveloped viruses requires an endosomal sorting complex required for transport (ESCRT), which is part of the cellular machinery used to form multivesicular bodies (MVBs). Tamai et al. demonstrated that Hrs, an ESCRT-0 component, is critical for the budding of $\mathrm{HCV}$ through the exosomal secretion pathway. ${ }^{41} \mathrm{Hrs}$ depletion caused reduced exosome production, which paralleled with the decrease of HCV replication in the host cell, and that in the culture supernatant. Sucrosedensity gradient separation of the culture supernatant of $\mathrm{HCV}$-infected cells revealed the co-existence of $\mathrm{HCV}$ core proteins and the exosome marker. Furthermore, both the core protein and an envelope protein of $\mathrm{HCV}$ were detected in the intraluminal vesicles of MVBs, suggesting that HCV secretion from host cells requires Hrs-dependent exosomal pathway in which the viral assembly is also involved.

Dreux et al. report that autophagy proteins (i.e., Beclin-1, Atg4B, Atg5, and Atg12) are proviral factors required for translation of incoming $\mathrm{HCV}$ RNA and, thereby, for initiation of $\mathrm{HCV}$ replication, but they are not required once infection is established. ${ }^{39}$ These results illustrate a previously unappreciated role for autophagy in the establishment of a viral infection and they suggest that different host factors regulate the translation of incoming viral genome and translation of progeny HCV RNA once replication is established. Tanida et al also demonstrated the decreases the production of infectious HCV particles by knockdown of autophagy-related genes. ${ }^{42}$ Atg7-knockdown decreases the amount of HCV replicon RNA. When Atg 7 protein was knocked down, the levels of intracellular $\mathrm{HCV}$ core, NS3, NS5A proteins, HCV mRNA and secreted albumin remained unchanged. However, the level of infectious HCV particles released in the medium was decreased by the Atg7-knockdown. Similar results were obtained when Beclin 1 was knocked down. These results suggested that autophagy contributed to the effective production of $\mathrm{HCV}$ particles.

The impact of the autophagy machinery on HCV infection was also reflected by indirect mechanism. Mizui et al. evaluate the autophagy of Huh-7 cells following the transfection of $\mathrm{HCV}$ replicon and then investigated the replication of $\mathrm{HCV}$ subgenomic replicon in response to treatment with lysosomal protease inhibitors or pharmacological autophagy inhibitor. ${ }^{43}$ The transfection of $\mathrm{HCV}$ replicon increased the number of autophagosomes. Pharmacological inhibition of autophagic proteolysis significantly suppressed expression level of HCV replicon. Silencing of autophagy-related genes by siRNA transfection significantly blunted the replication of HCV replicon. Treatment of replicon cells with chloroquine suppressed the replication of the $\mathrm{HCV}$ replicon. Furthermore, combination treatment of chloroquine to IFN $\alpha$ enhanced the antiviral effect of IFN $\alpha$ and prevented repropagation of HCV replicon. Protein kinase R (PKR) was activated in cells treated with IFN $\alpha$ but not with chloroquine. Incubation with chloroquine decreased degradation of long-lived protein leucine. This study suggest that the replication of HCV replicon utilizes machinery involving cellular autophagic proteolysis.

\section{DOWN-REGULATION OF INNATE IMMUNE RESPONSE BY HCV THROUGH UPR AND AUTOPHAGY INDUCTION}

Autophagy involves the sequestration of cytoplasmic content within a double-membraned autophagosome, and the fusion of the autophagosome with a lysosome to form an autolysosome for subsequent degradation. HCV proteins accumulate and often lead to ER stress and induction of the UPR. HCV-induced UPR may activate autophagy to support the virus life cycle.

$\mathrm{Ke}$ et al. reported that HCV induces the UPR, which in turn activates the autophagic pathway to promote HCV RNA replication in human hepatoma cells. ${ }^{44}$ Gene silencing or activation of the UPR-autophagy pathway activated or repressed, respectively, IFN- $\beta$ activation mediated by an HCV-derived pathogen-associated molecular pattern (PAMP), indicating that HCV may exploit the UPR-autophagy pathway to escape the innate immune response.

Shrivastava et al. also demonstrated the innate immune response could be enhanced in HCV-infected hepatocytes by knockdown of autophagy. Knockdown of beclin 1 (BCN1) or ATG7 in immortalized human hepatocytes (IHHs) inhibited HCV growth. BCN1- or ATG7-knockdown IHHs, when they were infected with $\mathrm{HCV}$, exhibited increased expression of interferon- $\beta$, 2 ', 5'-oligoadenylate synthetase 1 , interferon- $\alpha$, and interferon- $\alpha$-inducible protein 27 messenger RNAs of the interferon signaling pathways. A subsequent study demonstrated that HCV infection in autophagyimpaired IHHs displayed caspase activation, poly (adenosine diphosphate ribose) polymerase cleavage, and apoptotic cell death. The disruption of autophagy machinery in HCV-infected hepatocytes activates the interferon signaling pathway and induces apoptosis. Together, these results suggest that $\mathrm{HCV}$-induced 
autophagy impairs the innate immune response.

Estrabaud et al. demonstrated that HCV induces UPR, which in turn activates the autophagic pathway to promote HCV RNA replication. ${ }^{45}$ Further analysis revealed that the entire autophagic process was required to promote $\mathrm{HCV}$ RNA replication and that it did so by suppressing innate antiviral immunity. Gene silencing or activation of the UPR-autophagy pathway activated or repressed, respectively, IFN- $\beta$ activation mediated by an HCV-derived PAMP. ${ }^{46}$ Taken together, these results not only define the physiological significance of HCV-induced autophagy, but also shed light on the knowledge of host cellular responses upon $\mathrm{HCV}$ infection as well as on exploration of therapeutic targets for controlling $\mathrm{HCV}$ infection.

\section{REFERENCES}

1. Kudchodkar SB, Levine B. Viruses and autophagy. Rev Med Virol 2009;19:359-378.

2. Deretic V, Levine B. Autophagy, immunity, and microbial adaptations. Cell Host Microbe 2009;5:527-549.

3. He C, Klionsky DJ. Regulation mechanisms and signaling pathways of autophagy. Annu Rev Genet 2009;43:67-93.

4. Orvedahl A, Levine B. Eating the enemy within: autophagy in infectious diseases. Cell Death Differ 2009;16:57-69.

5. Shoji-Kawata S, Levine B. Autophagy, antiviral immunity, and viral countermeasures. Biochim Biophys 2009;1793:1478-1484.

6. Feldstein A, Kleiner D, Kravetz D, Buck M. Severe hepatocellular injury with apoptosis induced by a hepatitis $\mathrm{C}$ polymerase inhibitor. J Clin Gastroenterol 2009;43:374-381.

7. Dreux M, Chisari FV. Impact of the autophagy machinery on hepatitis C virus infection. Viruses 2011;3:1342-1357.

8. Sir D, Ann DK, Ou JH. Autophagy by hepatitis B virus and for hepatitis B virus. Autophagy 2010;6:4383-4388.

9. Coller KE, Berger KL, Heaton NS, Cooper JD, Yoon R, Randall G. RNA interference and single particle tracking analysis of hepatitis C virus endocytosis. PLoS Pathog 2009;5: e1000702.

10. Vivithanaporn $\mathrm{P}$, Maingat $\mathrm{F}$, Lin L-T, Na H, Richardson $\mathrm{CD}$, Agrawal B, et al. Hepatitis $\mathrm{C}$ virus core protein induces neuroimmune activation and potentiates human immunodeficiency virus-1 neurotoxicity. PLoS One 2010;5:e12856.

11. Sir D, Liang C, Chen WL, Jung JU, Ou JH. Perturbation of autophagic pathway by hepatitis C virus. Autophagy 2008;4:830-831.

12. Gondois-Rey F, Dental C, Halfon P, Baumert TF, Olive D, Hirsch I. Hepatitis $\mathrm{C}$ virus is a weak inducer of interferon alpha in plasmacytoid dendritic cells in comparison with influenza and human herpesvirus type-1. PLoS ONE 2009;4:e4319.

13. Arnaud N, Dabo S, Maillard P, Budkowska A, Kalliampakou $\mathrm{KI}$, Mavromara $\mathrm{P}$, et al. Hepatitis C virus controls interferon production through PKR activation. PLoS One 2010;5: e10575.

14. Dreux M, Gastaminza P, Wieland SF, Chisari FV. The autophagy machinery is required to initiate hepatitis $\mathrm{C}$ virus replication. Proc Natl Acad Sci USA 2009;106:14046-14051.

15. Jung JH, Park JH, Jee MH, Keum SJ, Cho MS, Yoon SK, et al. Hepatitis C virus infection is blocked by HMGB1 released from virus-infected cells. J Virol 2011;85:9359-9368.

16. Banerjee A, Ray RB, Ray R. Oncogenic potential of hepatitis C virus proteins. Viruses 2010; 2:2108-2133.

17. Osna NA, Thomes PG, Terrence M Donohue Jr. Involvement of autophagy in alcoholic liver injury and hepatitis $\mathrm{C}$ pathogenesis. World J Gastroenterol 2011;17:2507-2514.

18. Berger KL, Cooper JD, Heaton NS, Yoon R, Oakland TE, Jordan TX, et al. Roles for endocytic trafficking and phosphatidylinositol 4-kinase III alpha in hepatitis C virus replication. Proc Natl Acad Sci USA 2009;106:7577-7582.

19. Gastaminza P, Pitram SM, Dreux M, Krasnova LB, Whitten-Bauer $\mathrm{C}$, Dong J, et al. Antiviral stilbene 1,2-diamines prevent initiation of hepatitis C virus RNA replication at the outset of infection. J Virol 2011;85:5513-5523.

20. Ke PY, Chen SS. Autophagy: a novel guardian of HCV against innate immune response. Autophagy 2011;7:533-535.

21. Wang N, Liang Y, Devaraj S, Wang J, Lemon SM, Li K. Tolllike receptor 3 mediates establishment of an antiviral state against hepatitis C virus in hepatoma cells. J Virol 2009;83: 9824-9834.

22. Guévin C, Manna D, Bélanger C, Konan KV, Mak P, Labonté P. Autophagy protein ATG5 interacts transiently with the hepatitis $\mathrm{C}$ virus RNA polymerase (NS5B) early during infection. Virology 2010;405:1-7.

23. Blackham S, Baillie A, Al-Hababi F, Remlinger K, You S, Hamatake R, et al. Gene expression profiling indicates the roles of host oxidative stress, apoptosis, lipid metabolism, and intracellular transport genes in the replication of hepatitis $\mathrm{C}$ virus. J Virol 2010;84: 5404-5414

24. Sun J, Desai MM, Soong L, Ou JH. IFN- $\alpha / \beta$ and autophagy: tugof-war between HCV and the host. Autophagy 2011;7:1394-1396.

25. Ait-Goughoulte M, Kanda T, Meyer K, Ryerse JS, Ray RB, Ray R. Hepatitis $\mathrm{C}$ virus genotype 1a growth and induction of autophagy. J Virol 2008;82:2241-2249.

26. Su WC, Chao TC, Huang YL, Weng SC, Jeng KS, Lai MM. Rab5 and class III phosphoinositide 3-kinase Vps34 are involved in hepatitis C virus NS4B-induced autophagy. J Virol 2011;85:10561-10571.

27. Estrabaud E, De Muynck S, Asselah T. Activation of unfolded protein response and autophagy during $\mathrm{HCV}$ infection modulates innate immune response. J Hepatol 2011;55:1150-1153.

28. Kim HJ, Lee S, Jung JU.When autophagy meets viruses: a double-edged sword with functions in defense and offense. Semin Immunopathol 2010;32:323-341.

29. Chu VC, Bhattacharya S, Nomoto A, Lin J, Zaidi SK, Oberley $\mathrm{TD}$, et al. Persistent expression of hepatitis $\mathrm{C}$ virus non-structural proteins leads to increased autophagy and mitochondrial injury in human hepatoma cells. PLoS One 2011;6:e28551.

30. Thoen LF, Guimarães EL, Grunsven LA. Autophagy: a new player in hepatic stellate cell activation. Autophagy 2012;8:126-128.

31. Vescovo T, Romagnoli A, Perdomo AB, Corazzari M, Ciccosanti F, Alonzi T, et al. Autophagy protects cells from HCV-induced defects in lipid metabolism. Gastroenterology 2012;142:644-653.

32. Nishina S, Korenaga M, Hidaka I, Shinozaki A, Sakai A, Gondo $\mathrm{T}$, et al. Hepatitis $\mathrm{C}$ virus protein and iron overload induce hepatic steatosis through the unfolded protein response in mice. Liver Int 2010;30:683-692.

33. Ferraris P, Blanchard E, Roingeard P. Ultrastructural and biochemical analyses of hepatitis $\mathrm{C}$ virus-associated host cell membranes. J Gen Virol 2010;91:2230-2237.

34. Merquiol E, Uzi D, Mueller T, Goldenberg D, Nahmias Y, Xavier RJ, et al. HCV causes chronic endoplasmic reticulum stress leading to adaptation and interference with the unfolded protein response. PLoS One 2011;6:e24660.

35. Guévin C, Manna D, Bélanger C, Konan KV, Mak P, Labonté P. Autophagy protein ATG5 interacts transiently with the hepatitis $\mathrm{C}$ virus RNA polymerase (NS5B) early during infection. Virology 2010;405:1-7.

36. Desai MM, Gong B, Chan T, Davey RA, Soong L, Kolokoltsov AA, et al. Differential, type I interferon-mediated autophagic trafficking of hepatitis $\mathrm{C}$ virus proteins in mouse liver. Gastroenterology 2011;141:674-685.

37. Grégoire IP, Richetta C, Meyniel-Schicklin L, Borel S, Pradezynski F, Diaz O, et al. IRGM is a common target of RNA viruses that subvert the autophagy network. PLoS Pathog 2011;7: e1002422.

38. Sir D, Chen WL, Choi J, Wakita T, Yen TS, Ou JH. Induction of incomplete autophagic response by hepatitis $\mathrm{C}$ virus via the unfolded protein response. Hepatology 2008;48:1054-1061.

39. Dreux M, Chisari FV. Autophagy proteins promote hepatitis C virus replication. Autophagy 2009;5:1224-1225.

40. Taguwa S, Kambara H, Fujita N, Noda T, Yoshimori T, Koike K, et al. Dysfunction of autophagy participates in vacuole formation and cell death in cells replicating hepatitis $\mathrm{C}$ virus. J Virol 2011;85:13185-13194.

41. Tamai K, Shiina M, Tanaka N, Nakano T, Yamamoto A, Kondo $\mathrm{Y}$, et al. Regulation of hepatitis $\mathrm{C}$ virus secretion by the Hrsdependent exosomal pathway. Virology 2012;422:377-385.

42. Tanida I, Fukasawa M, Ueno T, Kominami E, Wakita T, Hanada 
K. Knockdown of autophagy-related gene decreases the production of infectious hepatitis $\mathrm{C}$ virus particles. Autophagy 2009;5:937-945.

43. Mizui T, Yamashina S, Tanida I, Takei Y, Ueno T, Sakamoto N, et al. Inhibition of hepatitis $\mathrm{C}$ virus replication by chloroquine targeting virus-associated autophagy. J Gastroenterol 2010;45:195-203.

44. Ke PY, Chen SS. Activation of the unfolded protein response and autophagy after hepatitis $\mathrm{C}$ virus infection suppresses innate antiviral immunity in vitro. J Clin Invest 2011;121:37-56
45. Shrivastava S, Raychoudhuri A, Steele R, Ray R, Ray RB. Knockdown of autophagy enhances the innate immune response in hepatitis C virus-infected hepatocytes. Hepatology 2011;53:406-414.

46. Grégoire IP, Richetta C, Meyniel-Schicklin L, Borel S, Pradezynski F, Diaz O, et al. IRGM is a common target of RNA viruses that subvert the autophagy network. PLoS Pathog 2011;7:e1002422. 